\title{
Stefan George et Karl Wolfskehl : le maître et le disciple?
}

Stefan George und Karl Wolfskehl: der Meister und der Jünger?

Stefan George and Karl Wolfskehl: the master and the disciple?

\section{Sonia Schott}

\section{(2) OpenEdition}

Journals

Édition électronique

URL : http://journals.openedition.org/ceg/351

DOI : $10.4000 /$ ceg. 351

ISSN : 2605-8359

Éditeur

Presses Universitaires de Provence

Édition imprimée

Date de publication : 5 avril 2017

Pagination : 89-102

ISBN : 979-10-320-0103-5

ISSN : 0751-4239

\section{Référence électronique}

Sonia Schott, "Stefan George et Karl Wolfskehl : le maître et le disciple ? », Cahiers d'Études

Germaniques [En ligne], 72 | 2017, mis en ligne le 05 octobre 2018, consulté le 26 novembre 2020.

URL : http://journals.openedition.org/ceg/351 ; DOI : https://doi.org/10.4000/ceg.351

Tous droits réservés 


\title{
Stefan George et Karl Wolfskehl \\ Le maître et le disciple?
}

\author{
Sonia SCHOTT \\ Université Toulouse Jean-Jaurès
}

Né en 1869 à Darmstadt dans une famille juive aisée, Karl Wolfskehl témoigne d'un vif goût pour la littérature qui le conduit à effectuer de brillantes études de germanistique. Après qu'il a rencontré Stefan George, le chef de file du symbolisme allemand, il publie à partir de 1894 des poèmes et des textes en prose dans Les Feuilles pour l'art (Die Blätter für die Kunst), la revue que Stefan George édite avec C.A. Klein, et il est également l'un des premiers membres du cercle autour de Stefan George, le George-Kreis, dont l'un des buts majeurs est d'encourager les vocations poétiques. Les revers économiques causés par la Première Guerre mondiale ruinent la famille de Wolfskehl. Le poète devient alors essayiste et collabore à divers journaux pour subvenir à ses besoins. En 1933 surviennent deux événements déterminants pour l'existence et la production de Wolfskehl : la mort de George et la victoire du nazisme en Allemagne. Juste après l'arrivée d'Hitler au pouvoir, le 31 janvier 1933, Wolfskehl part en exil pour l'Italie. En 1938, après que Mussolini a aligné sa politique sur l'antisémitisme du III Reich, Wolfskehl se voit contraint de s'exiler en Nouvelle-Zélande où il demeure jusqu'à sa mort en 1948. De 1933 à 1948, la problématique essentielle qui sous-tend les poèmes d'exil de Karl Wolfskehl, Juif et Allemand, est celle d'un sentiment de double appartenance. Sa poésie se fait alors écho d'une profonde crise existentielle et alimente, par un processus dialogique, une réflexion sur la volonté du Dieu du judaïsme $(Y H W H)$ quant à la condition humaine, proposant par-là une herméneutique de la souffrance ${ }^{1}$ comparable à celle du livre de Job dans la Bible.

De cette esquisse biographique, on retient que la vocation poétique de Karl Wolfskehl connaît deux moments décisifs : la rencontre avec Stefan George en 1891 et l'exil à partir de 1933. L'objet de cet article porte sur le premier point: l'influence de Stefan George sur la poésie d'avant l'exil de Karl Wolfskehl et la question de savoir si, et en quel sens, George fut pour lui un modèle. Avant le tournant de 1933, son œuvre se décline en trois recueils, à savoir ULAIS (1897), Gesammelte Dichtungen (1903) - dans lequel les poèmes d'ULAIS sont repris - et Der Umkreis (1927). Malgré la publication tardive de Der Umkreis,

1. Formule empruntée à Manfred Koch, "Rilke und Hölderlin. Hermeneutik des Leids ", Blätter der Rilke Gesellschaft, ${ }^{\circ}$ 22, 1999, p. 91-105. 
la plupart des poèmes du recueil ont été rédigés entre 1897 et 1912 . L'avis des contemporains est partagé quant à la poésie de Karl Wolfskehl : le 30 avril 1897, Stéphane Mallarmé, qui a lu ULAIS, écrit à Stefan George qu'il considère Wolfskehl comme un " admirable poète ${ }^{2}$ ". En outre, un germaniste consacre dès 1928 son travail de thèse à l'étude de la poésie de ce dernier ${ }^{3}$. À propos des Gesammelte Dichtungen, Stefan Zweig en revanche déplore la médiocrité de Wolfskehl, qui se laisse défınir seulement par rapport à George et dont les vers manquent de musique, de forme et de profondeur ${ }^{4}$.

Selon Wolfskehl lui-même, le rapport à George est constitutif de son identité poétique. Il n'hésite pas à dire dans son article en prose "Begegnung mit Stefan George $^{5}$ " que c'est la lecture des poèmes de George qui donne un sens à sa vie, et que George représente pour lui une norme. Bien après la dissolution du cercle et la mort de George (4.12.1933), en dressant son autoportrait dans une lettre d'exil, Wolfskehl affirme que sa grande fierté est d'être un "épigone " de

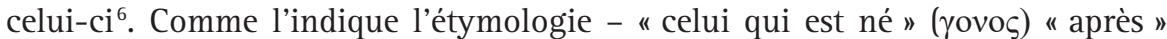
$(\dot{\varepsilon} \pi \mathrm{l})$ - et la mythologie qui s'y rattache ${ }^{7}$, le terme d'épigone sert à désigner un successeur, au sein d'un parti, d'une école littéraire ou philosophique. Ce terme se distingue néanmoins de son corrélat latin "disciple " dans la mesure où il est le plus souvent employé avec une visée péjorative. L'épigone serait alors celui qui ne fait qu'imiter le maître, qui n'innove pas. Dire d'un artiste qu'il est l'épigone d'un autre artiste ou d'un mouvement, c'est souligner le caractère à la fois non originel et non original de son œuvre, qui s'apparente à une pâle copie

2. Lettre de Mallarmé à George du 30 avril 1897, Valvins. Cité d'après: Manfred Schlösser, Karl Wolfskehl 1869-1969. Leben und Werk in Dokumenten, Darmstadt, Agora Verlag, 1969, p. 82-83.

3. Edwin Landau, Karl Wolfskehl. Stilkritische Untersuchungen seiner Lyrik, Hellerau, Jakob Hegner Verlag, 1928.

4. "... ich vermisse viel in den Versen von Karl Wolfskehl: [...] Musik, Plastik und Tiefe. Vor allem aber eine Persönlichkeit. Man wird Karl Wolfskehl nie anders als mit Stefan George definieren können. ", Stefan Zweig, "Die um Stefan George ", Das literarische Echo, 1.11.1903. Cité d'après M. Schlösser, Leben, p. 36.

5. "Gegen Ende des Jahres 1892 lernte ich in Giessen kennen, was bis dahin von Stefan George erschienen war: seine drei ersten Gedichtbände (Hymnen, Pilgerfahrten, Algabal) [...] mir widerfuhr, was nicht jedem Leben zuteil wird, nie sich im selben Dasein wiederholt: ich wusste von dem Augenblick an, wohin ich gehöre, was in mir Norm sei. [...] [es] war mir mit einem Male klar geworden, was mit mir, mir selber los sei, wohin ich selber gehöre, was von da ab mein Leben zu bedingen habe. Obwohl ich natürlich genug und übergenug in Knaben- und Pubertätsgedichten und ähnlichem mir geleistet hatte und mein erstes wirkliches Gedicht erst ein gutes Jahr später in den $>$ Blättern $<$ erschien, ist doch damals der Dichter in mir geboren worden. [...] Plötzlich war die Welt zwar nicht $>$ vollkommen $<$, aber sie hatte einen Sinn erhalten, man wusste, warum man da war und zu welchem Ende ", in Margot Ruben (dir.), Karl Wolfskehl. Briefe und Aufsätze. München 1925-1933, Hamburg, Claassen Verlag, 1966, p. 185-186. Première publication, in Die literarische Welt IV, 1928, p. 29.

6. Cf. la lettre de Karl Wolfskehl à Siegfried Guggenheim du 19.9.1947 : "Karl Wolfskehl, dessen großer Stolz es ist, ein „George-Epigone“ zu sein ", in Cornelia Blasberg (dir.), " Du bist allein, entrückt, gemieden " Karl Wolfskehls Briefwechsel aus Neuseeland, 1938-1948, t. I, Darmstadt, Luchterhand Literaturverlag, 1988, p. 657.

7. Pierre Grimal, Dictionnaire de la mythologie grecque et romaine, Paris, PUF, 1951, p. 141-142. Les Épigones sont les descendants des Sept contre Thèbes. 
plutôt qu'à une création. On comprend que Wolfskehl use des guillemets pour se qualifier lui-même d'épigone, car il est le premier à condamner la copie servile: Il n'hésite en effet pas à reprocher à son jeune ami Friedrich Gundolf d'imiter George $^{8}$, soulignant par cette même critique le caractère autonome de la création poétique ou la singularité de chaque auteur.

Du point de vue de l'histoire littéraire, le mouvement initié en Allemagne par George, le symbolisme, se définit par une réaction au naturalisme et aux épigones d'un romantisme finissant ${ }^{9}$. Pour les symbolistes, il s'agit d'innover et non plus d'imiter, d'opérer dans l'écriture une révolution, excluant tout asservissement $d u$ langage à une autre fin que lui-même ${ }^{10}$. La dynamique propre au(x) cercle(s) de Stefan George favorisait néanmoins la distinction entre, d'une part, le maître, George lui-même, qui a eu tendance, comme la recherche s'accorde à le montrer, à cultiver une posture de plus en plus hiératique ${ }^{11}$, et ses "disciples ".

Pour caractériser l'œuvre de jeunesse de Wolfskehl, convient-il alors de souscrire à l'avis de Mallarmé ou de Zweig? Est-il un "admirable poète " ou n'est-il qu'un pâle ersatz de George? Les rapports entre maître et disciples inhérents à la dynamique du cercle de Stefan George ont-ils une incidence sur la production poétique de Wolfskehl? Eu égard au rapport étroit entre George et Wolfskehl, il est immanquable que s'effectuent des transferts entre les œuvres des deux poètes. Ces échanges se font-ils alors sur le mode de l'imitation du modèle, voire de la copie?

8. « Im ersten Jahr seiner Begegnung mit George imitiert Gundolf George so eng, dass sein Freund und Mentor Karl Wolfskehl bremsend auf ihn einwirkt: "Bewundern im Thun umgesetzt heißt nicht nachahmen, fremdes herübernehmen, sondern im Eigensten auf eigenste Weise der fremden Größe entgegenzueilen!” ", in Gunilla Eschenbach, Imitatio im George-Kreis, Berlin/ New York, De Gruyter, 2011, p. 94.

9. Cf. Cornelia Blasberg, "George-Kreis »: "George hatte solche Erwartungen geweckt durch seine radikale Absage an die Felix-Dahn- und Gartenlaube-Leser der Gründerzeit, die mit der pathetisch-nationalistischen oder sentimental-neuromantischen Lyrik in epigonaler Formensprache zufriedengestellt werden konnten. ", in Dan Diner (dir.), Enzyklopädie jüdischer Geschichte und Kultur. Im Auftrag der Sächsischen Akademie der Wissenschaften zu Leipzig, Stuttgart/Weimar, 2012, p. 426-430, (citation p. 427).

10. " der name dieser veröffentlichung sagt schon zum teil was sie soll: der kunst besonders der dichtung und dem schriftum dienen, alles staatliche und gesellschaftliche ausscheidend. Sie will die GEISTIGE kunst auf grund der neuen fühlweise und mache - eine kunst für die kunst ». Blätter für die Kunst, série 1, vol. I, cité in Georg Peter Landmann, Der George-Kreis, Köln/Berlin, Kiepenheuer\&Witsch, 1965, p. 15.

11. Jürgen Egyptien, " Die "Kreise” ", in Achim Aurnhammer, Wolfgang Braungart, Stefan Breuer, Ute Oelmann (dir.), Stefan George und sein Kreis. Ein Handbuch, Berlin/Boston, De Gruyter, 2012, vol.1, p. 365-407. 


\section{L'admiration de Wolfskehl pour George}

L'admiration voire la vénération de Wolfskehl pour George est certaine. Ainsi lui dédicace-t-il ses trois recueils de poèmes $U L_{A I S}{ }^{12}$, Gesammelte Dichtungen ${ }^{13}$ et Der Umkreis ${ }^{14}$, qui parurent au moment de leur collaboration, avant l'exil de Wolfskehl et le décès de George en 1933. En guise de préface aux Gesammelte Dichtungen figure en outre le texte en prose "Der Priester vom Geiste ${ }^{15}$ " qui est un l'éloge par Wolfskehl de Stefan George ${ }^{16}$. Le recueil se clôt sur le poème Der Meister ${ }^{17}$ dont le dernier vers donne la parole au maître, qui s'adresse à ses disciples pour leur rappeler son rôle décisif: "Euch dank ich mein WISSEN: mir danket den WEG ! " Dans ce vers, le parallèle entre "WISSEN " et " WEG ", qui est souligné par l'emploi des capitales d'imprimerie, est ici exemplaire de la conception que Wolfskehl avait quant au rapport d'émulation entre maître et disciple. Au maitre incombe en effet une fonction maïeutique (il montre le chemin aux apprentis poètes) tandis que le second apporte de la matière et des connaissances, sur le mode d'un "transfert de savoir " (Wissenstransfer). Outre les trois dédicaces, l'œuvre de jeunesse de Wolfskehl est traversée de références à George. En témoigne, dans la forme, le fait que le poète utilise le même code typographique que son maitre pour la conception de ses livres, à savoir le renoncement aux majuscules, le point en haut, le choix d'un papier de grande qualité et le recours aux enluminures de Melchior Lechter, artiste graphiste faisant également partie du George-Kreis. Ces recours au Code-Zitat ${ }^{18}$ sont autant de manières pour Wolfskehl de professer son attachement à George.

Les allusions thématiques à la personne du " maître " parcourent également l'ensemble de l'œuvre de jeunesse : ainsi Manfred Durzak ${ }^{19}$ interprète-t-il le poème

12. «Ulais: [...] Titel des ersten eigenen Gedichtbandes von Wolfskehl im Verlag der Blätter für die Kunst (Berlin 1897). Widmung: „An Stefan George“, Titelblatt von Melchior Lechter ", in Karlhans Kluncker (dir.), Karl und Hanna Wolfskehl: Briefwechsel mit Friedrich Gundolf 1899-1931, vol. 1, Amsterdam, Castrum Peregrini Presse, 1977, 2e éd., p. 257.

13. "An Stefan George ", in Karl Wolfskehl, Gesammelte Werke, vol. I. Dichtungen. Dramatische Dichtungen, Hamburg, Claassen Verlag, 1960, p. 7.

14. « Dis. Magistro. Amicis ", in ibid., p. 65.

15. Le texte fut publié initialement sans les Blätter für die Kunst en janvier 1896. Les éditeurs des œuvres complètes de Wolfskehl ont pris le parti de reproduire ce texte dans le volume consacré à la prose. Nous citons d'après l'original: Karl Wolfskehl, Gesammelte Dichtungen, Berlin, Bondi, 1903, p. 5-8.

16. "Wolfskehls Prosamythe ,Der Priester vom Geiste“ von 1895 geht in der Sakralisierung Georges - implizit auch seines eigenen Dichtungverständnisses - noch weiter ", in Friedrich Voit, Karl Wolfskehl. Leben und Werk im Exil, Göttingen, Wallstein Verlag, 2005, p. 28.

17. Wolfskehl, Werke, p. 60.

18. Cf. Dieter Lamping, "Intertextualitätsanalyse ", in Thomas Anz, Handbuch Literaturwissenschaft, vol. II. Methoden und Theorien, Stuttgart/Weimar, Metzler, 2007, p. 204-224. On peut y lire: "Man kann Stile zitieren, also spezifische Verwendungsweisen eines gegebenen Zeichensystems, die für bestimmte Epochen, Gruppen oder Individuen typisch sind.» (p. 210).

19. Manfred Durzak, " Der junge Karl Wolfskehl. Zu den frühen Gedichten in den Blättern für die Kunst ", in Elke-Vera Kotowski, Gert Mattenklott (dir.), "O dürft ich Stimme sein, das Volk zu rütteln! "Leben und Werk von Karl Wolfskehl (1869 - 1948), Hildesheim/Zürich/New York, Olms, 2007, p. 121-131. 
Die Erlösung ${ }^{20}$, qui consacre la célébration du retour d'un prince ardemment attendu par sa suite, comme un sonnet poétologique à la gloire de George. Dans le poème Berufung ${ }^{21}$, paru dans Der Umkreis, le thème de la vocation est abordé : comme l'a souligné Ute Oelmann ${ }^{22}$, la relation entre l'instance lyrique et l'allocutaire du poème - Karl Wolfskehl et Stefan George - est allégorisée par le recours au Nouveau Testament: "Ich bin dein knecht ich will dein Petrus sein ". On sait que Pierre était à la fois le disciple et le successeur de Jésus, le fondement de son Église: Tout au long de son œuvre, Wolfskehl se considèrera toujours comme celui à qui incombe la mission de pérenniser l'œuvre de son maître.

Même après le décès de Stefan George, la figure du maître reste prédominante pour Wolfskehl. Dans le poème Das Lebenslied. An die Deutschen ${ }^{23}$, Wolfskehl retrace sa biographie ainsi que celle de sa lignée pour interroger le destin de la symbiose judéo-allemande. George est la figure tutélaire du poème, car Wolfskehl cite trois extraits ${ }^{24}$ de l'œuvre poétique de George sans citer personne d'autre. Ces trois citations dont les mots-clés sont " geist " et " erneuung " consacrent la doctrine de George, tout en étant resémantisées à l'aune de l'exil enduré par Wolfskehl. Dans ce même poème, la référence à George se fait non seulement sur le mode de la citation mais également sur celui de l'allusion puisque les vers 17, 61 et 67 - "Wo ich bin ist deutscher Geist " - font écho à l'Allemagne secrète (Geheimes Deutschland ${ }^{25}$ ) que George évoque dans Das neue Reich. En outre, alors que l'ensemble du poème, comme l'indique le titre, s'adresse aux Allemands qui sont apostrophés par le pronom "ihr " ou par un "Teut " emblématique, les quatre derniers vers de l'ultime strophe avant l'envoi changent d'allocutaire et, après une énallage, s'adressent uniquement à George :

Morgens Meister, Stern der Wende

Hat Ihn lang mein Sang genannt:

Sohn der Kür, Bote der Sende

Bleib ich, Flamme, Dir Trabant!

Wolfskehl effectue ici un acte d'allégeance et consacre l'apothéose de George, la flamme dont il n'est que le satellite et l'humble serviteur. L'image même qu'il emploie pour assurer de sa fidélité est un emprunt littéral à son maître ${ }^{26}$.

20. Wolfskehl, Werke, p. 13.

21. Ibid., p. 100-101. Première publication in Blätter für die Kunst, 1910.

22. Ute Oelmann, " "ich will dein Petrus sein". Karl Wolfskehl und Stefan George ", in Kotowski/ Mattenklott, Leben, p. 41-52.

23. Wolfskehl, Werke, p. 216-219. Poème rédigé à partir de 1934, première publication: Zürich, Origo Verlag, 1947.

24. Après le titre: " Die Weltzeit die wir kennen schuf der geist (Stefan George) ". Après le premier sous-titre: "Kein stern und kein jahr/ Vernichtet den geist/ Allmächtig so wahr/ Er noch wundert und preist (Stefan George) ". Après le second sous-titre: " Nur aus dem fernsten her kommt die erneuung (Stefan George) ".

25. Stefan George, Das neue Reich (= Sämtliche Werke in 18 Bänden herausgegeben von Ute Oelmann, Band IX), Stuttgart, Klett-Cotta, 2001, p. 45-49.

26. "Wer je die flamme umschritt/ Bleibe der flamme trabant! ", in Stefan George, Der Stern des Bundes (= Sämtliche Werke in 18 Bänden herausgegeben von Ute Oelmann, Band VIII), Stuttgart, Klett-Cotta, 1993, p. 84. 
Cette citation exprime non seulement la révérence personnelle mais elle permet également de réaffirmer un principe poétologique ${ }^{27}$ : en s'inscrivant ainsi dans la lignée de George, Wolfskehl réaffirme la consécration de son existence au service de la poésie.

\section{L'autonomie de Wolfskehl}

Bien qu'à l'instar de la relation "Flamme/ Trabant", les métaphores que Wolfskehl emploie pour évoquer sa relation avec George renvoient toujours à une relation hiérarchisée entre dominant et dominé, cela caractérise davantage les relations interpersonnelles entre les deux hommes - qui relèvent du domaine de la psychologie et de la biographie - que la poétique des deux auteurs. Stefan George lui-même souligne le caractère singulier de la poésie de Wolfskehl ${ }^{28}$, de même que les études littéraires consacrées au George-Kreis s'attachent à reconnaître l'originalité de Wolfskehl. Ainsi Manfred Durzak commence-t-il son article sur les épigones au sein du George-Kreis en déclarant d'emblée que cela ne peut concerner Wolfskehl ${ }^{29}$, de même que Gunilla Eschenbach ${ }^{30}$, qui propose une typologie de l'imitation dans le cercle de Stefan George, souligne l'autonomie du poète.

À l'instar du vers "Euch dank ich mein WISSEN/ Mir danket den WEG ", soulignons que Wolfskehl était tout à fait conscient des apports qu'il pouvait fournir à son maître. On peut signaler à cet égard que Claude David montre dans sa thèse que le messianisme de Wolfskehl influence les thèmes et le mode d'écriture du Septième anneau de George ${ }^{31}$. La proximité entre les poèmes des deux auteurs

27. «Dienst an der Poesie [...] Alles bloss Subjektive muss aber zurückgedrängt werden. Stefan George verpflichtet sich diesem Gedanken, wenn er sein Leben lang an die Aufgabe erinnert, die das Subjekt zu leisten hat: an das Amt, das angemessen auszuüben ist, an "die flamme", deren "trabant" man zu bleiben hat [...] ". Wolfgang Braungart: " Poetik, Rhetorik, Hermeneutik ", in Aurnhammer, Braungart, Breuer, Oelmann (dir.), Stefan George und sein Kreis. Ein Handbuch, vol. 2, p. 518-519.

28. Cf. la lettre de Stefan George à Ida Koblenz de septembre 1895: " ich sehe in ihm den einzigen unter den > Jungen< der die forderung begriffen nicht aus einem erzählchen heraus sondern aus rausch und rhythmus heraus zu schaffen. [...] W. bringt neues ", in Georg Peter Landmann (dir.), Stefan George. Ida Coblenz. Briefwechsel, Stuttgart, Klett-Cotta Verlag, 1983, p. 58.

29. " denn neben George sind zumindest zwei Stimmen von dichterischen Eigenkraft und Rang in den Blättern vertreten, nämlich Wolfskehl, und jenem überlegen und George ebenbürtig, Hofmannsthal, der freilich nicht im eigentlichen Sinne zum George-Kreis zu rechnen ist ", in Manfred Durzak, "Epigonenlyrik. Zur Dichtung des George-Kreises ", in Jahrbuch der deutschen Schillergesellschaft 13 (1969), Stuttgart, Alfred Kröner Verlag, 1969, p. 482-529 (citation p. 484-485).

30. "Nur wenige Beiträger seiner Zeitschrift Blätter für die Kunst bezeichnet George gegenüber Hofmannsthal als "urbedingt" und "einzig", so etwa Ludwig Klages oder Karl Wolfskehl. Diese sogenannte "Urgeister" imitieren nicht, sondern sind dazu befähigt, ihre individuellen Anlagen unabhängig von der Anleitung durch andere zur Vollendung zu treiben. ", in Eschenbach, Imitatio, p. 2.

31. "À sa mobilité d'esprit, à son inquiétude, à son goût de la nouveauté et de l'expérience, Wolfskehl joint une tendance messianique que l'on pourra, si l'on veut, dire juive, un espoir toujours en éveil, l'attente d'événements proches destinés à transformer le monde tout à 
est parfois très marquée et nous aimerions maintenant illustrer par un exemple comment l'imitation peut procéder d'un phénomène d'émulation féconde.

\title{
Un poème de Wolfskehl en regard de celui de George
}

\author{
Vogelschau ${ }^{32}$
}

Weisse schwalben sah ich fliegen

Schwalben schnee- und silberweiss

Sah sie sich im winde wiegen

In dem winde hell und heiss.

Bunte häher sah ich hüpfen

Papagei und kolibri

Durch die wunder-bäume schlüpfen

In dem wald der Tusferi.

Grosse raben sah ich flattern

Dohlen schwarz und dunkelgrau

Nah am grunde über nattern

Im verzauberten gehau.

Schwalben seh ich wieder fliegen

Schnee- und silberweisse schar

Wie sie sich im winde wiegen

In dem winde kalt und klar!

Blondel III ${ }^{33}$

Weisse weisse blüte tauen

Lenz und lied,

Lauten laden, hehre frauen

Sehnend von dem söller schauen,

Lied und lenz.

Müde müde glieder beben,

Lied und leid,

Von dem söller schwalben schweben,

Bergen ferne sich in reben,

Leid und lied.

Schwarze schwarze linnen laben,

Lust und lied,

Schöner frauen stolzer knaben

Schlanke hände ihn begraben

Lied und lust.

coup et à délivrer d'une angoisse séculaire. Wolfskehl est une âme de prophète. Sans doute ces sentiments sont-ils appuyés et entretenus par beaucoup d'érudition et très consciemment rattachés à une tradition. La part de l'intelligence est grande, et c'est elle qui se forge des armes contre elle-même. Mais ce messianisme ne peut être négligé ; il éclaire toute une part du Septième Anneau: c'est la part de Wolfskehl. ", in Claude David, Stefan George. Son œuvre poétique, Paris / Lyon, IAC, Bibliothèque de la société des études germaniques, 1952, p. 197-198.

32. Stefan George, Hymnen Pilgerfahrten Algabal, in Sämtliche Werke in 18. Bänden, éd. par Ute Oelmann, vol. II, Stuttgart, Klett-Cotta Verlag, 1987.

33. Wolfskehl, Werke, p. 15. 
Manfred Schlösser écrit:

Die starke Abhängigkeit Karl Wolfskehls im Formalen wie Metaphorischen von dem 1892 entstandenen Schlussgedicht des Albagal „Vogelschau“ ist ziemlich eindeutig, doch die Gesamtatmosphäre ist geheimnisvoller, dunkler, Wolfskehlischer ${ }^{34}$.

Le parallèle entre le poème Vogelschau de George, rédigé au plus tard fin 1891 et publié en 1892 à la fin du recueil Algabal et Blondel III de Wolfskehl (paru dans Les Feuilles pour l'art en 1894 (deuxième livraison, quatrième cahier) a été établi par Manfred Schlösser, qui souligne à la fois la dépendance ("Abhängigkeit ") et l'autonomie ("Wolfskehlischer ") de Wolfskehl, sans entrer davantage dans les détails. Notre analyse tentera de montrer dans quelle mesure le poème de Wolfskehl reproduit celui de George et comment il s'en démarque.

Le titre choisi par Wolfskehl est une allusion à Schubert ${ }^{35}$ et non à George. La figure de l'hirondelle constitue un net emprunt de Wolfskehl à George: ainsi le huitième vers de Blondel, "Von dem söller schwalben schweben ", fait-il écho au premier vers de Vogelschau, "weisse schwalben sah ich fliegen ". Wolfskehl a en outre recours à la même symbolique que George: les deux poèmes font allusion à la pratique des augures ou ornithomancie, selon laquelle le vol des oiseaux est révélateur du destin. Eu égard au sort qu'annoncent les oiseaux, on peut déceler une première différence entre les deux poèmes dans la mesure où le mouvement du poème chez Wolfskehl se fait vers la mort. "Blondel " meurt à la fin du poème (" ihn begraben") alors que chez George le retour des hirondelles dans la dernière strophe après l'évocation des corbeaux suggère métaphoriquement une renaissance.

Si l'on observe le choix des mots qui composent le poème Blondel III, on constate que malgré l'emprunt littéral de " schwalben ", Wolfskehl développe sa musicalité propre. Chez George, la consonne chuintante de "schwalbe " trouve un écho dans "schnee " et "schar " (v. 2 et 14), et le premier vers joue des assonances en " a " " ei ". Ces dernières seront prolongées dans l'ensemble de la strophe par "silberweiss " (v. 2), "sah" (v. 1 et 3) et " heiss " (v. 4). Wolfskehl compose sa propre musique allitérative par la paronomase entre "schwalben " et "schweben " (v. 8) auquel feront ensuite écho "schwarz ", "schön " et " schlank " (v. 11, 13 et 14). C'est davantage de Brentano que de George que Wolfskehl se rapproche dans la mesure où l'on ne peut manquer d'entendre Lieb und Leid im leichten Leben ${ }^{36}$ dans le jeu allitératif voire paronomastique sur l'amour et la souffrance qui préside à la conclusion de chacune des strophes du poème " Leid /Lied/ Lust/ Lenz ".

En ce qui concerne le sens des poèmes, l'écart entre clarté et obscurité est caractéristique de la différence entre les poétiques de Wolfskehl et de George. De la lecture du poème de George, on retire une impression de clarté : les références

34. Schlösser, Leben, p. 77.

35. Franz Schubert, Blondel zu Marien, D. 626, 1818 (première publication en 1842).

36. Clemens Brentano, Sämtliche Werke und Briefe, vol. 2,1, Gedichte 1801-1806, Stuttgart, Kohlhammer Verlag, p. 24-25. 
à des impressions visuelles - "Vogelschau " "sah ich " " seh ich " - ainsi que la syntaxe claire des phrases y contribuent, qui rendent à l'instar de la dernière strophe, le vers clair et précis " hell und klar ". Une telle netteté des images ne se retrouve pas chez Wolfskehl. Une des causes en est que les strophes ne sont pas construites sur le modèle thème / rhème - c'est-à-dire l'évocation des oiseaux et la caractérisation de leur vol -, mais repose sur une structure et une syntaxe plus lâches, dont le principe est l'accumulation paratactique de sujets indéfinis. Tel un tableau impressionniste, le poème wolfskehlien se joue du flou pour devenir évocation d'un état d'âme. Wolfskehl est ce faisant un fervent symboliste dans la mesure où son texte témoigne d'une volonté de poésie pure débarrassée non seulement des fonctions de communication mais également de dénotation ${ }^{37}$. Les mots se dissolvent dans la musique du vers, sont combinés tant pour leur valeur sonore que descriptive. C'est ainsi que la répétition immédiate d'un même adjectif au début de chaque strophe (weisse weisse / müde müde / schwarze schwarze) montre que le poète use, comme le dit Baudelaire " de la langue et de l'écriture, prises comme opérations magiques, sorcellerie évocatoire ${ }^{38}$ ".

On retiendra du poème de George le froid qu'il dégage, tout en faisant allusion à son indéniable portée poétologique ${ }^{39}$. De celui de Wolfskehl, on rappellera le caractère onirique, eu égard aux nombreux flous de la description. Un esprit de fin de siècle émane de la mort qui paraît plutôt douce et sereine, et la gestuelle d'enterrement rappelle le maniérisme du Jugendstil. On notera avec intérêt que George relève chez Wolfskehl dès la publication de ULAIS en 1897 le caractère flou et incertain de son écriture et lui conseille de changer cela à travers la métaphore du lever de brouillard :

Was soll ich Ihnen darüber neues sagen der ich alle Ihre gedichte (mit 2-3 ausnahmen) schon kenne und geschätzt habe. Es liegt über ihnen ein weicher nebel hie und da noch dicht und wenn sie ihn eines tages heben so werden wir mit Staunen die gefilde noch reicher noch holder erblicken! ${ }^{40}$

37. "Le vers qui de plusieurs vocables refait un mot total, neuf, étranger à la langue et comme incantatoire, achève cet isolement de la parole [...] et vous cause cette surprise de n'avoir ouï jamais tel fragment ordinaire d'élocution, en même temps que la réminiscence de l'objet baigne dans une neuve atmosphère ", in Stéphane Mallarmé, CEuvres complètes, vol. II, édition établie par Bertrand Marchal, Paris, Pléiade Gallimard, 2003, p. 213.

38. Charles Baudelaire, "Fusées XI", in CEuvres complètes, vol. I, édition établie par Claude Pichois, Paris, Pléiade Gallimard, 1975, p. 658.

39. Allusion poétologique explicitée par Wolfgang Braungart, "Poetik, Rhetorik, Hermeneutik " in Stefan George und sein Kreis. Ein Handbuch, p. 503.

40. Lettre de George à Wolfskehl du 16.2.1897, in "Von Menschen und Mächten " Stefan George - Karl und Hanna Wolfskehl. Der Briefwechsel 1892-1933, herausgegeben von Birgit Wägenbaur und Ute Oelmann im Auftrag der Stefan George Stiftung, München, Beck, 2015, p. 180. 


\section{Caractéristiques de l'art poétique de Wolfskehl}

À la lecture des recueils respectifs de George et Wolfskehl, il apparaît que les poèmes de George relèvent davantage d'une beauté apollinienne tandis que les vers de Wolfskehl, tout en participant d'une esthétique symboliste ${ }^{41}$, sont à la mesure de sa nature extatique et dionysiaque ${ }^{42}$. Afın d'étayer cette dernière thèse, nous proposons une brève analyse du poème Zarathustra, qui est paradigmatique dans sa configuration de l'art poétique wolfskehlien.

Zarathustra fut publié dans Les Feuilles pour l'art en mai 1901, peu de temps après le décès de Nietzsche qui est un philosophe important tant pour le George-Kreis ${ }^{43}$ que pour la pensée de Wolfskehl ${ }^{44}$. Dans les œuvres complètes de Wolfskehl, le poème figure dans le premier cycle du recueil qui porte le titre de Nänie. Ce titre renvoie au poème de Schiller Auch das Schöne muss sterben et fait également référence à l'Antiquité, puisque la nénie (nenia en latin) est le chant funèbre à la louange d'un personnage de marque, entonné chez les Romains par des pleureuses à gages. Procédant à la fois du deuil et de l'éloge, ce poème est un " tombeau ", que John E. Jackson caractérise comme un "poème consacré à "créer" la renommée de celui ou celle dont il porte mémoire ${ }^{45}$ " et auquel Wolfskehl a été sensibilisé par la lecture de Mallarmé. Le poème passe du personnage (Zarathustra) à l'auteur (Nietzsche), et de la vie vécue par ce dernier à la postérité laissée dans l'histoire de la pensée. On peut parler à cet égard de "poème-portrait ${ }^{46}$ " (Porträtgedicht).

\section{ZARATHUSTRA}

Stumm throntest du am torweg jahre jahre,

An dir vorbei lief leiden lust und lärm,

Am torweg drinn du mit dem zwerg geflüstert

Ehe du dich entrückt...nun sassest du

Tief innen dein blick und dein wissen tief innen.

41. Paul Hoffmann, Symbolismus, München, UTB, Fink Verlag, 1987.

42. Paul Hoffmann, "Wolfskehls Dichtung im Entwicklungzusammenhang der modernen Lyrik ", in Paul Klussmann, Karlhans Kluncker et al., Karl Wolfskehl Kolloquium. Vorträge. Berichte. Dokumente, Amsterdam, Castrum Peregrini Presse, 1983, p. 61-85.

43. Heinz Raschel, Das Nietzsche-Bild im George-Kreis. Ein Beitrag zur Geschichte der deutschen Mythologeme, Berlin/New York, de Gruyter, 1984, (= Monographien und Texte zur NietzscheForschung, vol. 12).

44. Cf. la lettre de Wolfskehl à Greta Hort du 9.8.1939: "Weiterhin wurde mein geistiges Gesicht von früh auf durch Nietzsche bestimmt ", in Karl Wolfskehl, Zehn Jahre Exil. Briefe aus Neuseeland.1938-1948, édité par Margot Ruben, Darmstadt / Heidelberg, Lambert Schneider Verlag, 1959, p. 58.

45. John E. Jackson, Mémoire et Création poétique, Paris, Mercure de France, 1992, p. 255.

46. "La configuration énonciative traditionnelle du poème-portrait, conçu à la fin du 18e siècle en signe d'hommage à un artiste vivant ou défunt repose sur une distinction de personne entre le sujet lyrique et l'allocutaire qui trouve son expression dans la structure dialogique du poème, fondé sur l'adresse lyrique du sujet écrivant à un "tu". ", in Nadia Lapchine, Poésie et histoire dans l'œuvre tardive d'Erich Arendt (1903-1984), t. I, Paris, L'Harmattan, 2003, p. 201. Cf. également: Eckart Kleßmann, Lyrische Porträts, Stuttgart, Reclam Verlag, 1991, p. 15-18. 


\begin{abstract}
Das höchste leben sass reglos und sann Gebannt vom eignen ruhbereiten sehnen. Mein ist die wahl! Du wähltest, einsamkeit Hielt dich umfangen -goss ihrem trunkenen Ein stetes abendglühen um die schläfe. Zur flamme lodert nun dein glühn empor Die dich Gebahrten raubend dich uns gibt.
\end{abstract}

Le subtil jeu d'écho entre les sonorités du titre et celles du premier vers laissent entrevoir au lecteur que Wolfskehl a faite sienne l'attitude mallarméenne consistant à "creuser le vers ${ }^{47}$ ". La lettre " $t$ ", dont le nom "Zarathustra " fournit deux occurrences, trouve en effet son prolongement dans l'allitération en dentales sur laquelle s'ouvre le poème, "stumm thronest du ", de même que le son " $\mathrm{u}$ " qui fait également partie du nom "Zarathustra " se retrouve dans le pronom personnel "du " et dans "stumm ". On note en outre que la voyelle " a " répétée par trois fois dans "Zarathustra " résonne dans la répétition " jahre jahre ». En ayant recours à ce procédé de répétition paratactique, Wolfskehl joue non seulement avec le sens de son expression (" des années des années ") mais il porte également une attention toute particulière à la matière-même du mot car la répétition du " a " long dans " jahre jahre " souligne le caractère lancinant de l'épreuve de Zarathoustra qui à la fois patient et impassible.

Le poète accomplit le même travail d'orfèvre sur les sons au vers suivant, qui introduit une allitération, "lief leiden lust und lärm ". C'est ici un procédé d'harmonie imitative qui se fait jour car la succession ininterrompue de la consonne liquide " 1 " souligne le passage en autant de vagues successives de la souffrance (leiden), du désir (lust) et du vacarme (lärm). La composition de ces deux premiers vers est savamment agencée par Wolfskehl, qui fait figurer en leur extrémité les antonymes " stumm " et " lärm " afin de rendre encore davantage sensible au lecteur l'opposition entre Zarathoustra et le monde. La sagesse de Zarathoustra le pousse à ne pas être accaparé par le monde et à se retirer en lui-même. Le cinquième vers, dont l'unité est subtilement soulignée par l'assonance en " i ", manifeste de par l'agencement des mots le retour sur soi qui y est évoqué. Wolfskehl effectue ce faisant une sémantisation de la forme de son poème. En effet, le chiasme issu de la répétition " tief innen " en début et en fin de vers met en valeur le parallélisme de construction entre " dein wissen " et " dein blick ». Cet encadrement crée un espace intérieur dans le vers qui symbolise l'intériorité de Zarathoustra et rend visible le retrait évoqué dans le vers précédent: " Ehe du dich entrückt ".

Si certes le genre traditionnel du poème-portrait se caractérise par l'allocution, Wolfskehl a recours à divers procédés de variations qui permettent d'éviter l'écueil de la monotonie. En effet les vers 1 à 5 étaient écrits à la deuxième personne, constituant donc une adresse directe à Zarathoustra, mais le $6^{\mathrm{e}}$ vers opère un changement de perspective. Le sujet du vers est "das höchste leben " qui repose sur un double procédé: une énallage, d'une part, puisqu'on passe de la deuxième à la troisième personne, et une métonymie d'autre part, le terme de

47. Lettre de Stéphane Mallarmé à Henri Cazalis du 28 avril 1866 (Tournon), in Stéphane Mallarmé, Euvres complètes, t. I, p. 696. 
"leben " tenant lieu de principe vital à l'homme, Zarathoustra, qui est évoqué à travers elle. On passe alors d'un poème dialogique à un poème-récit. À l'image du souverain impassible succède désormais celle de l'homme qui songe. Ainsi la paronomase entre les verbes " sass et sann " joue-t-elle de l'effet d'écho comme rapprochement notionnel car Zarathoustra est ici présenté " assis et réfléchissant ", dans la position typique du penseur moderne, telle que l'exemplifie la célèbre statue de Rodin. Le procédé paronomastique permet à Wolfskehl de relier non seulement sitzen et sinnen, mais également sinnen et sehnen, en une triade riche de sens: Certes de l'extérieur, Zarathoustra est présenté comme détaché du monde (" entrückt "), assis (" sass ") et immobile (" reglos"). La paronomase "sasssann-sehnen " fait cependant passer de l'immobilité apparente à l'intranquillité latente, comme l'évoque au $7^{\mathrm{e}}$ vers l'oxymore " ruhbereiten sehnen ". Le huitième vers rompt avec le mode du récit et dramatise le poème par l'introduction du discours direct: " Mein ist die wahl " procède à nouveau de l'énallage et le lecteur comprend que ce sont les paroles de Zarathoustra. Il ne s'agit pas, pour autant que l'on sache, d'une citation littérale de Nietzsche mais d'une création de Wolfskehl. L'emphase introduite par la mise en relief de l'adjectif possessif " mein " en début de phrase trouve son pendant dans la dérivation lexicale " wahl du wähltest " au centre du vers, les deux procédés subtilement maniés par le poète permettant de souligner la détermination de Zarathoustra. S'il va sans dire que la solitude de Zarathoustra est l'un des traits les plus marquants de l'œuvre de Nietzsche ${ }^{48}$, l'apport de Wolfskehl se situe non dans le fond mais dans la forme. Le huitième vers du poème tend en effet vers la perfection formelle en ceci qu'il opère un continuum sonore entre le cri de Zarathoustra "mein ist die wahl " qui est bâti sur la succession vocalique " ei " et " a " et l'objet de ce choix, à savoir la solitude, puisque le mot " einsamkeit " repose sur l'assonance entre " ei " et " a ". On remarquera en outre que dans ce vers, Wolfskehl joue sur la discordance entre mètre et syntaxe, puisque " einsamkeit ", bien que séparé par un signe de ponctuation du verbe " wählen ", se trouve néanmoins de par le contre-rejet à la place du COD. Il convient également de lire qu' " einsamkeit " est sujet de " hielt ", ce en quoi l'enjambement ${ }^{49}$ opère une transition remarquable, puisqu'auparavant sujet en majesté, le « tu " apostrophé devient maintenant objet : " Einsamkeit hielt dich umfangen ". La construction verbale avec un participe passé passif comme attribut du complément d'objet direct souligne en effet que c'est la solitude qui est désormais maîtresse du jeu, et non plus le penseur, comme si ce dernier avait affaire à plus puissant que lui. Alors qu'auparavant Zarathoustra semblait délivré des passions humaines, le vers 8 souligne de manière contrastive qu'il est

48. "Mein ganzer Zarathustra ist ein Dithyrambus auf die Einsamkeit, oder, wenn man mich verstanden hat, auf die Reinheit. ", in Friedrich Nietzsche, Kritische Gesamtausgabe, éd. par Giorgio Colli et Mazzino Montinari, VI, 3, Berlin, de Gruyter, 1969, p. 274. [Ecce homo] Henning Ottman (dir.), Nietzsche Handbuch. Leben-Werk-Wirkung, Stuttgart/Weimar, Metzler, 2011, p. 218 (article " Einsamkeit ").

49. On trouve une autre analyse détaillée de cet enjambement, in Paul Hoffmann, Das religiöse Spätwerk Karl Wolfskehls, Dissertation, Wien Universitätsbibliothek, 1958. (thèse non publiée dont une copie se trouve aux archives littéraires de Marbach am Neckar). 
comme prisonnier de sa solitude. Le vers 9 confirme cette impression, puisque Zarathoustra y est à nouveau objet, présenté grâce à un participe passé passif ("trunkenen "). Le jeu sur les pronoms possessifs montre aussi la métamorphose de Zarathoustra et le caractère paradoxal de cette solitude. En effet, Zarathoustra, en possession de son destin " mein ist die Wahl ", est devenu possédé "ihrem trunkenen ".

Le tour de force de Wolfskehl est de réussir à fait advenir dans la métrique le changement qui est évoqué par le poème. De même que l'impassibilité du sage se mue en ivresse solitaire, le vers devient de plus en plus agité. Ainsi, vers et phrase coïncident-ils au vers 1 et 2 . Les vers 3 et 4 témoignent encore d'une relative stabilité car le vers 3 est composé de la proposition principale, tandis que la première partie du quatrième vers est constitué par la subordonnée temporelle. Les transitions entre les quatrième, cinquième, sixième et septième vers se font sans heurt, car elles sont axées sur des phénomènes d'appositions réguliers. En revanche, c'est précisément au moment où l'impassibilité zoroastrienne se transforme en extase que les vers 8, 9 et 10 font l'objet d'enjambements manifestes.

Dans les deux derniers vers du poème, la métaphore du feu qui est filée (" glühn ", " flamme "), n'est guère originale et se trouve dans le Zarathustra de Nietzsche ${ }^{50}$, qui exploite ensuite dans Ecce homo ${ }^{51}$ la métaphore du feu de manière bien plus subversive que Wolfskehl ne le fait ici. C'est dans la concision du $12^{\text {e }}$ et dernier vers que résident à la fois sa beauté et sa difficulté. En cette séquence verbale typique de son écriture, le poète évoque la fugacité de la vie humaine, en condensant d'une part trois verbes déclinés sur trois modes distincts et d'autre part en usant du jeu d'oxymores entre le don (" gebären ") et le vol (" rauben ") de la vie, qui fait également une allusion au caractère éminemment prométhéen de l'entreprise nietzschéenne.

L'évocation de la gloire posthume de Nietzsche en fin de poème et de son importance pour les contemporains procède d'une visée encomiastique des plus traditionnelles pour un tombeau. À cet égard, le lecteur moderne sera peutêtre davantage charmé par la réflexion poétologique et le caractère novateur de la dissonance suggérée par le reproche à peine voilé de George à Nietzsche, qui resémantise un passage de l'introduction à la Naissance de la tragédie:

Nietzsche: Sie hätte singen sollen, diese « neue Seele » - und nicht reden! ${ }^{52}$

George: Und wenn die strenge und gequälte stimme

Dann wie ein loblied tönt in blaue nacht

Und helle flut - so klagt: Sie hätte singen

Nicht reden sollen diese neue seele! ${ }^{53}$

50. " als der Greis zu Zarathustra spricht: „Damals trugst du deine Asche zu Berge: willst du heute dein Feuer in die Täler tragen? Fürchtest du dich nicht des Brandstifters Strafen? ", in Friedrich Nietzsche, Kritische Gesamtausgabe VI. 1, p. 6.

51. "Ich bin kein Mensch, ich bin Dynamit. ", in Friedrich Nietzsche, Kritische Gesamtausgabe VI. 3, p. 363.

52. Friedrich Nietzsche, Kritische Gesamtausgabe, III. 1, p. 9.

53. Stefan George, Poésies complètes, traduites de l'allemand, présentées et annotées par Ludwig Lehnen. Édition bilingue, Paris, Éditions de la Différence, 2009, p. 390. 
Les hommages de Wolfskehl et de George permettent de constater d'une part que Wolfskehl n'a pas seulement pris George pour modèle, mais également Nietzsche, et de l'autre que George lui-même n'hésitait pas à se considérer - peut-être en mésinterprétant certains passages de l'œuvre du philosophe - comme un disciple (quelque peu critique) de Nietzsche.

\section{Conclusion}

Les distinctions entre modèle et copie, innovation et imitation paraissent peu opératoires pour caractériser le rapport littéraire entre Wolfskehl et George, qui est davantage fondé sur la réciprocité. Certes George a influencé Wolfskehl mais Wolfskehl a également influencé George. Partant, le jeu d'échos intertextuels entre les deux auteurs relève non d'un rapport entre modèle et épigone mais de l'émulation, c'est-à-dire d'une dynamique féconde qui enrichit leurs poétiques respectives.

L'une des causes majeures de l'admiration du " disciple " pour son " maittre " est à rapporter au fait que c'est grâce à Stefan George que Karl Wolfskehl a pris la mesure de l'exigence formelle que requiert l'écriture poétique. Le raffinement et l'originalité avec lesquels il travaille les sonorités de ses vers montrent qu'il est un poète symboliste accompli. À cet égard, les enjambements abrupts et les tournures brachylogiques qui contribuent à l'opacité du sens sont autant de procédés qui caractérisent son art poétique. 